\title{
Operant conditioning permits voluntary, noninvasive measurement of blood pressure in conscious, unrestrained baboons (Papio cynocephalus)
}

\author{
DANIEL S. MITCHELL \\ Department of Bioengineering, Southwest Research Institute, San Antonio, Texas 78284 \\ HERMAN S. WIGODSKY \\ Department of Pathology, University of Texas Health Science Center, San Antonio, Texas 78284
}

H. HERBERT PEEL

Department of Bioengineering, Southwest Research Institute, San Antonio, Texas 78284

and

\author{
TIMOTHY A. MCCAFFREY \\ Department of Cardiopulmonary Disease, Southwest Foundation for Research and Education \\ San Antonio, Texas 78284
}

\begin{abstract}
This report describes a computerized apparatus and procedure for completely automated noninvasive measurement of systolic, diastolic, and mean arterial blood pressure in conscious, unrestrained nonhuman primates. We used a specially constructed, cage-mounted oscillometric blood pressure instrument and operant reward conditioning methods to train adult baboons (Papio cynocephalus) to submit voluntarily to frequent self-initiated determinations of their blood pressures in the absence of a human operator/observer. Details concerning the operant training procedure and illustrative blood pressure data are presented. The accuracy of the method was evaluated by comparing the noninvasive blood pressure readings with simultaneously taken direct measurements obtained by means of chronically indwelling arterial catheters.
\end{abstract}

Most methods of measuring the arterial blood pressure (BP) of nonhuman primates have various disadvantages that limit their usefulness in studies involving simultaneous measurement of behavioral and cardiovascular responses and in long-term experiments that require repeated BP determinations in large numbers of subjects. Direct measurement by means of percutaneous arterial puncture requires the use of anesthetics, which may produce marked and unpredictable alterations in BP, and can result in occlusion or other damage to the arteries used. Chronic preparations involving surgically implanted catheters also may damage the arteries; necessitate chairing, radiotelemetry, or tethering procedures; and are tedious and expensive to establish and maintain. Such preparations also may restrict the normal behavioral repertoire of experimental subjects.

Development of this method was supported by Specialized Center of Research in Arteriosclerosis Grant HL-19362 from the National Heart, Lung, and Blood Institute of the National Institutes of Health. Requests for reprints should be addressed to Daniel S. Mitchell, Behavioral Sciences Section, Department of Bioengineering, Southwest Research Institute, 6220 Culebra Road, San Antonio, Texas 78284.
Automated noninvasive methods that use the ultrasonic Doppler principle or those that rely on electronic processing of Korotkoff sounds permit frequent measurements and eliminate the risk of arterial injury, but they demand precise placement of transducer elements and are susceptible to acoustic and mechanical noise. The accuracy in nonhuman primates of these automated indirect methods has been questioned (e.g., McMahan, Wigodsky, \& Moore, 1976). The standard auscultatory technique sometimes has been used with monkeys, but reliable detection of Korotkoff sounds in nonhuman primates by human listeners is not always possible (Smith \& Ansevin, 1957), and the contact with a human observer required by this method may alter the animal's BP substantially. Both automated and manual indirect methods require sedation or physical restraint unless the subjects are specially trained to accept the procedures.

This report describes a computerized apparatus and procedure for automated noninvasive measurement of systolic, diastolic, and mean arterial BP in conscious, unrestrained baboons trained to participate voluntarily in the measurement process. 


\section{BLOOD PRESSURE APPARATUS}

\section{Oscillometric Method}

Of the various noninvasive methods for measuring BP, we selected the oscillometric technique as the most suitable for use with conscious, unrestrained baboons. In the oscillometric method, an air-filled bladder connected to a pressure transducer serves both as an occlusion cuff and as a sensor to detect pressure pulsations ("oscillations") transmitted from the underlying artery. Oscillometry requires no microphones or other transducers that demand precise placement and low-noise environments. It permits accurate readings to be taken from the forearm (Mitchell, Peel, Wigodsky, \& Morris, 1980), and it can be completely automated.

\section{System Overview}

The apparatus consists of an occlusion cuff assembly, a cuff pressurization and depressurization system, a pressure transducer, and a signal processing module. These devices are connected to a Data General Nova 3/12 computer that controls all system functions, logs the pulsatile and nonpulsatile components of cuff pressure, and computes systolic, diastolic, and mean arterial BP. The computer inflates and deflates the cuff by operating a set of solenoid valves connected to a regulated source of compressed air. The cuff assembly, pressurizationdepressurization system, and pressure transducer form part of a portable subassembly termed the "cage unit."

During a measurement sequence (trial), the cuff is inflated to approximately 50 torr above systolic BP, held at pressure for $2 \mathrm{sec}$, then deflated linearly to about 25 torr below diastolic BP, after which residual cuff pressure is vented. Cuff pressure data are recorded during the deflation phase, which lasts approximately $35 \mathrm{sec}$. Each complete trial requires about $40 \mathrm{sec}$. All readings are taken from the animals' upper forearms.

\section{Pressure Transducer and \\ Signal Processing Module}

The pressure transducer (National Semiconductor Corporation Model LX1704GZ) contains an integral amplifier that produces an effective sensitivity of 155 torr $/ \mathrm{V}(3 \mathrm{psi} / \mathrm{V})$ over the range \pm 755 torr $( \pm 15 \mathrm{psig})$. The output of the transducer feeds two subcircuits in the signal processing module. One subcircuit consists of an operational amplifier used for zero suppression and scaling. Its output is a dc signal proportional to the nonpulsatile component of cuff pressure. In the other subcircuit, two operational amplifiers are connected to form an active band-pass filter with gain that extracts the pulsatile component of the pressure signal and amplifies it to a usable level. The filter has a bandwidth of 1 to $45 \mathrm{~Hz}(-3 \mathrm{~dB})$, rolls off at $60 \mathrm{~dB} /$ decade, and has a gain of 130 in its band-pass region. The output of the band-pass filter is connected to a peak detection circuit that measures the amplitude of each pressure pulse and updates a sample-and-hold circuit. The latter produces a dc level proportional to the maximum amplitude of the pressure pulse. Each time the sample-and hold circuit is updated, a ready signal notifies the computer that data are to be taken. The computer, via A/D converters, then reads and stores the nonpulsatile cuff pressure value and the corresponding pulse amplitude captured by the sample-and-hold circuit. In the absence of noise artifacts, one pressure pulse occurs with each heartbeat.

\section{Cage Unit}

Figure 1 illustrates the cage unit that makes it possible to obtain BP readings from conscious, unrestrained animals. In the description that follows, capital letters within parentheses refer to corresponding features in the figure.

A $36 \times 80 \mathrm{~cm}$ sheet of 8 -gauge aluminum forms the front panel (A) of the cage unit and provides a rigid mounting surface for all other subassemblies and components. A $7.62-\mathrm{cm}$-diam hole aligned with a similar-sized hole in the front of the animal's cage gives access to the occlusion cuff assembly, which attaches to the rear surface of the front panel. A $28-\mathrm{V}$ dc power supply (B), a BRS/LVE (Tech Serv, Inc., Beltsville, Maryland 20705) primate reward pellet dispenser (C), and a set of three solenoid valves (D) also are mounted on the front panel. One solenoid valve controls the admission of compressed air to inflate the cuff, a second governs its normal slow deflation through a low-pressure regulator and needle-valve orifice $(E)$, and the third permits immediate and complete venting of cuff pressure. The valves are connected to an independent safety device that causes instant depressurization of the entire system in the event of a computer failure or loss of electrical power. A $5 \times 7.6 \mathrm{~cm}$ Plexiglas window (F) in the front panel allows the animal to view a pair of red and green cue lights (G).

The occlusion cuff assembly, shown in the inset of Figure 1, consists of three principal subunits: a cuff mounting subassembly $(\mathrm{H})$, a primate response level $(\mathrm{J})$, and a telescoping extender $(\mathrm{K})$.

Two $15.25 \mathrm{~cm} \times 15.25 \mathrm{~cm} \times 8$ gauge parallel aluminum end plates, each containing a $7.62-\mathrm{cm}$-diam hole and held $12.75 \mathrm{~cm}$ apart by $.95-\mathrm{cm}$-diam spacers (L), form the framework of the cuff mounting subassembly. A flexible "sleeve" made of lightweight double-knit fabric bridges the gap between the holes and is attached to the facing (inner) surfaces of the end plates by means of retaining rings and hose clamps (M). A human childsize BP cuff (N) wraps around the outside of the fabric sleeve. One hose of the cuff attaches to the pressurizationdepressurization system, and the other connects to the pressure transducer (P). The standard Velcro fasteners supplied on the cuff, coupled with the flexibility of the double-knit fabric sleeve, permit rapid adjustments in the diameter of the cuff to accommodate forearms of different circumferences.

The response lever (BRS/LVE) mounts behind a sheet 


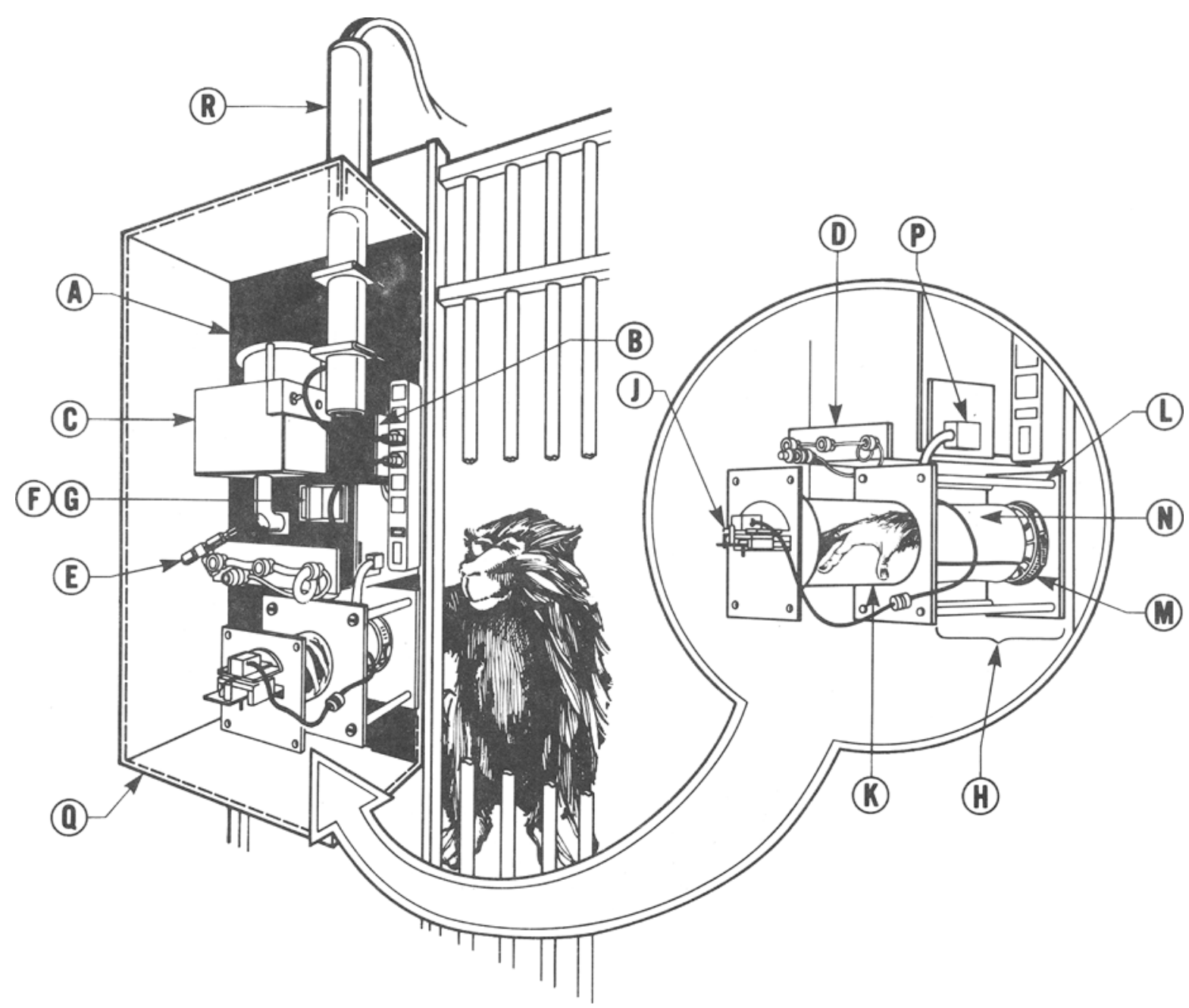

Figure 1. Artist's illustration of the cage-mounted components of the noninvasive BP measurement apparatus. Inset: detail of occlusion cuff assembly.

metal shroud that limits access to fingertips only. Fingertip activation of the lever eliminates artifacts in the cuff pressure/pulse data caused by flexing of major muscle groups in the forearm.

The telescoping extender consists of an $11.5-\mathrm{cm}$ length of $7.62-\mathrm{cm}$ (outer diameter) acrylic tubing (wall thickness $=6.35 \mathrm{~mm}$ ) that slides into a 5 -cm-long section of $7.62-\mathrm{cm}$ (inner diameter) aluminum pipe (wall thickness $=6.35 \mathrm{~mm}$ ). The acrylic tube is attached directly over the $7.62-\mathrm{cm}$ hole in the outer end plate of the cuff mounting subassembly, and the aluminum pipe is connected to the response lever subassembly. The extender thus forms a hollow cylinder or "barrel," with the response lever centered in its bore at the end farthest from the animal's cage. The telescoping feature provides a quick and efficient method of adjusting the distance between the cuff and the response lever subassemblies. Once adjusted, the desired distance can be fixed by a metal hose clamp that applies radial compression to the aluminum pipe (compression is facilitated by a set of longitudinal slots machined in the pipe).
Arrangement of the components of the cuff assembly is such that, in order to reach the response lever, the animal must extend its arm first through the cuff subassembly and then through the telescoping extender. This action automatically positions the occlusion cuff around the forearm. Fine adjustments in cuff position are accomplished by manipulating the length of the telescoping extender. A sliding metal door permits the experimenter to block the opening to the cuff assembly.

When closed, a hinged sheet metal cover (Q) completely encloses the cage unit to protect its components from damage by the animals. As a further precaution against mischief, all electrical leads and the compressed air line enter and exit through a $60-\mathrm{cm}$ length of $5-\mathrm{cm}$ (inner diameter) PVC pipe (R). A pair of tab-into-slot hangers and a spring-loaded latch allow the cage unit to be attached or removed from the cage in a matter of seconds. The complete unit weighs about $10 \mathrm{~kg}$.

\section{Blood Pressure Computation}

As cuff pressure decreases from occlusive levels, 
the oscillometric pulsations produced in the bladder first increase in amplitude, pass through a maximum, then decline, and ultimately disappear as cuff pressure falls below diastolic BP. Prior experiments conducted in our laboratory (Mitchell et al., 1980) and studies reported by others have shown that systolic BP is related to the region of most rapid increase in pulsation amplitude, mean BP corresponds to the region of maximum pulsation amplitude, and diastolic BP is associated with the region of most rapid decline in pulsation amplitude (Bard, 1956; Best \& Taylor, 1961; Deitenbeck, 1977; Flack, Hill, \& McQueen, 1915; Geddes, Moore, Garner, Rosborough, Ross, \& Amend, 1970; Gley \& Gomez, 1931; Looney, 1978; Pachon \& Fabre, 1921; Posey, Geddes, Williams, \& Moore, 1969; Ramsey, 1977). Our system uses a specially developed set of computational algorithms and corresponding computer programs that identify the three key regions by comparing slopes of successive overlapping segments of the pulse amplitude curve. No human judgments enter into the determination of the BP values.

\section{OPERANT TRAINING}

A distinctive feature of this method of measuring BP is its reliance on operant reward conditioning techniques to train the animals to participate voluntarily in the measurement process. Standard shaping procedures are used to establish a behavioral sequence in which the animal extends its forearm into the cuff assembly (hence through the occlusion cuff), depresses the lever to initiate a trial, and maintains arm position throughout the measurement sequence. Successful completion of a trial results in the delivery of a $750-\mathrm{mg}$ banana-flavored pellet followed by an obligatory intertrial interval of about $60 \mathrm{sec}$. Releasing the lever before the normal completion of a trial (caused, for example, by withdrawal of the arm) causes immediate venting of cuff pressure, withholding of reward, and initiation of a 120 -sec timeout, during which the apparatus cannot be activated. A green cue light signifies that the apparatus is operative, and a red cue light provides response feedback to the animal by coming on and staying on as long as the lever remains depressed.

The computer controls training of the animals, except for the first one or two $30-\mathrm{min}$ sessions of magazine training and response lever shaping, which are accomplished by the experimenter's manual activation of the pellet dispenser. Initially, the animal's task is simplified by removing the cuff and extender sections from the cuff assembly and mounting the response lever directly against the front panel of the cage unit. This causes the lever to protrude slightly into the cage, where it can be reached with minimal arm extension. In addition, the lever depression time (hold time) required to earn a reward is set at about .l sec. Once reliable leverpressing has been established, the hold time requirement is increased gradually over sessions of training, and the animal is forced to extend its arm farther and farther into the cuff assembly by inserting a series of increasingly long sections of acrylic tubing $(7.62-\mathrm{cm}$ inner diameter) between the lever and the front panel. Hold times are increased in .25- to .5-sec steps until times of 3-4 $\mathrm{sec}$ are achieved; thereafter, 2- to 5 - $\mathrm{sec}$ increments are possible. Increases in arm extension are made in $2.5-\mathrm{cm}$ increments.

Installation of the BP cuff and the telescoping extender is not attempted until the animal achieves full forearm extension and 30- to 40-sec hold times. Inflation of the cuff begins at 25 torr and increases over sessions of training in increments of about 25 torr. A temporary $50 \%-60 \%$ reduction in hold time for the first few sessions of cuff inflation appears to facilitate the animals' acceptance of the procedure. Except for hypertensive subjects, a maximum inflation level of 200 torr usually is adequate to obtain accurate $B P$ readings.

Aside from the obligatory intertrial interval, the initiation and pacing of trials is completely controlled by the subjects. Suitable motivation is ensured by withholding the animal's daily rations until after the sessions of training or BP measurement.

\section{ILLUSTRATIVE DATA}

The subjects were three male and one female adult baboons (Papio cynocephalus) who had no prior experience with noninvasive BP measurement procedures or with laboratory operant conditioning tasks. The individually caged animals were estimated to be 5.5 to 7.0 years old, ranged in weight from 14.5 to $23.5 \mathrm{~kg}$, and received ad-lib quantities of fresh water and Purina monkey chow daily, supplemented with fresh fruit several times each week.

All four baboons were trained successfully to cooperate in the BP measurement procedure. The number of training sessions, which averaged $60 \mathrm{~min}$ each, ranged from 35 to 51 with a mean of 43 .

Upon completion of training, a typical $60-\mathrm{min}$ session of monitoring yielded 10-20 BP measurements, which often occurred in discrete groupings of two to four closely spaced trials. The intermittent pacing of trials may have been modulated by the increase and decline of ischemic discomfort caused by frequent, repeated inflations of the occlusion cuff. All readings were taken with a 9-cm-wide cuff, a size that fulfilled the limb diameter/cuff width ratio needed for optimal results with auscultatory BP methods (Kirkendall, Burton, Epstein, \& Freis, 1967).

The number of noninvasive measurements of $\mathrm{BP}$ obtained from the four trained baboons ranged from 177 to 195 . The overall averages and standard deviations of each animal's systolic, diastolic, and mean arterial BPs appear in Table 1. Figure 2 shows a series of average systolic and diastolic BPs taken on consecutive days 
Table 1

Average Noninvasive Blood Pressure Readings from Four Conscious, Unrestrained Baboons

\begin{tabular}{|c|c|c|c|c|c|c|c|c|}
\hline \multirow[b]{3}{*}{ Baboon } & \multirow[b]{3}{*}{ Sex } & \multirow{3}{*}{$\begin{array}{c}\text { Number of } \\
\text { Readings }\end{array}$} & \multicolumn{6}{|c|}{ Blood Pressure (in Torr) } \\
\hline & & & \multicolumn{2}{|c|}{ Systolic } & \multicolumn{2}{|c|}{ Diastolic } & \multicolumn{2}{|c|}{ Mean } \\
\hline & & & Mean & SD & Mean & SD & Mean & SD \\
\hline$X-767$ & $\mathbf{M}$ & 195 & 124.1 & 13.6 & 76.2 & 12.6 & 96.8 & 14.0 \\
\hline$X-934$ & $\mathrm{~F}$ & 186 & 142.5 & 14.5 & 94.9 & 11.9 & 103.5 & 13.0 \\
\hline $\mathrm{X}-3239$ & $\mathrm{M}$ & 177 & 126.9 & 10.7 & 77.1 & 8.9 & 87.4 & 11.6 \\
\hline$X-3362$ & $\mathbf{M}$ & 183 & 131.7 & 20.2 & 84.9 & 15.9 & 100.1 & 19.3 \\
\hline
\end{tabular}

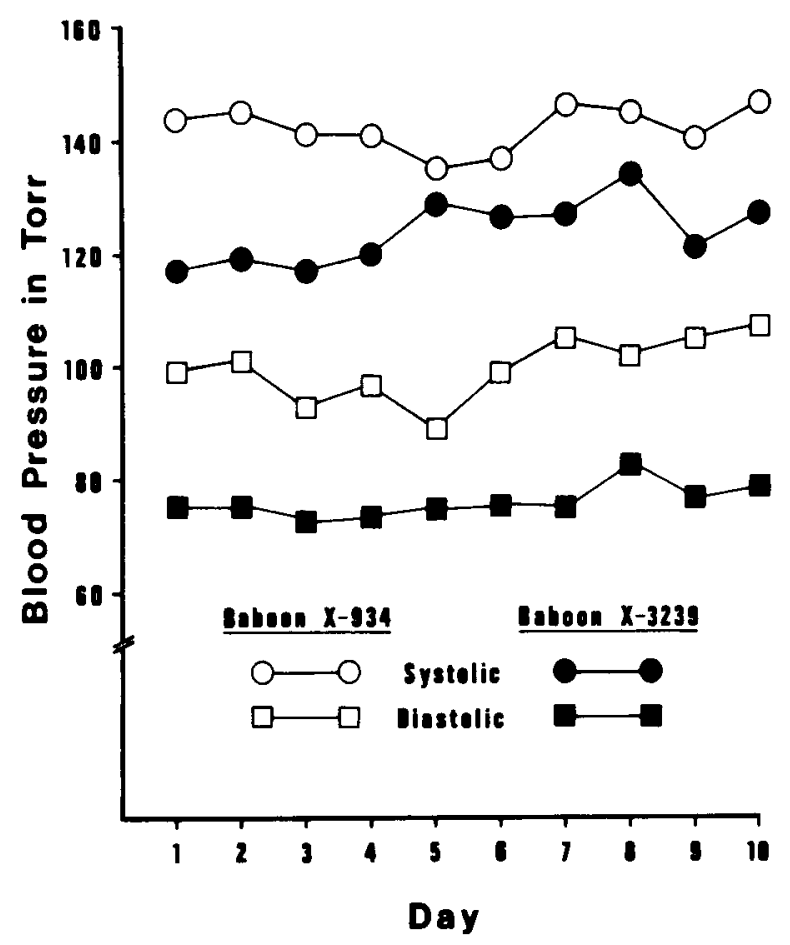

Figure 2. Systolic and diastolic noninvasive BP measurements taken on consecutive days from two conscious, unrestrained baboons.

from two of the four animals. Each data point represents the mean of $10-20$ readings. Despite the day-to-day variations in $\mathrm{BP}$, our method consistently differentiated the animal with moderately elevated systolic and diastolic pressures (X-934) from her normotensive partner.

\section{ACCURACY OF BLOOD PRESSURE READINGS}

In order to evaluate the accuracy of the noninvasive readings, a tethering system similar to that described by Byrd (1979) was used to obtain continuous direct recordings of arterial BP from two trained baboons. This system consisted of a lightweight fiberglass backpack, held on the animal by thoracic bands and shoulder straps, and a flexible stainless steel umbilical cord connecting the backpack to an electrocannular slip-ring in the top of the cage. An indwelling catheter inserted retrograde into the abdominal aorta via the internal iliac artery and connected to a pressure transducer in the backpack permitted beat-by-beat measurement of BP in conscious animals. The tether imposed no constraints on the activity and position of the subjects within their home cages.

One hundred twenty-five and 139 simultaneous noninvasive and direct measurements of $\mathrm{BP}$ were made on the two tethered baboons during 10 consecutive sessions of testing. After adjusting for hydrostatic differences in pressure, error scores were computed by subtracting each noninvasive reading from its corresponding direct measurement. Averaging error scores over animals and trials revealed mean errors of -5.1 torr in the noninvasive measurements of systolic $\mathrm{BP},+.3$ torr for diastolic BP, and -11.9 torr for mean BP. The lower average mean BP measured by the noninvasive method is consistent with the fact that mean pressures measured in the brachial and radial arteries typically are 5.8 torr lower than mean aortic pressures (Selkurt, 1966). Separate repeated-measures analyses of variance of the simultaneously taken noninvasive and direct readings of systolic, diastolic, and mean BP revealed no statistically significant differences as a function of method of measurement or session of testing (all ps $>.15$ ). The Method by Session interaction terms also failed to achieve significance, indicating that the accuracy of the noninvasive readings remained stable over days of testing. The overall accuracy of the noninvasive readings in conscious animals compared favorably with that observed in earlier tests of the oscillometric method in anesthetized baboons (Mitchell et al., 1980).

Figure 3 shows 10 successive simultaneous noninvasive and direct measurements of BP taken from one of the trained and tethered baboons. With the exception of mean pressure on Trial9, the noninvasive determinations tracked with good precision the normal fluctuations in BP shown in the direct measurements. The 33-torr error in the noninvasive reading of mean pressure on Trial 9 probably was due to animal movement or related artifacts that occurred during the measurement process. Data from tethered animals indicate that about $15 \%$ of the noninvasive readings of systolic, diastolic, and mean BP will differ by more than 20 torr from simultaneously taken direct readings. Such errors usually are easy to detect and eliminate by comparing the suspect value with other readings that precede and follow it. Averaging several successive determinations 


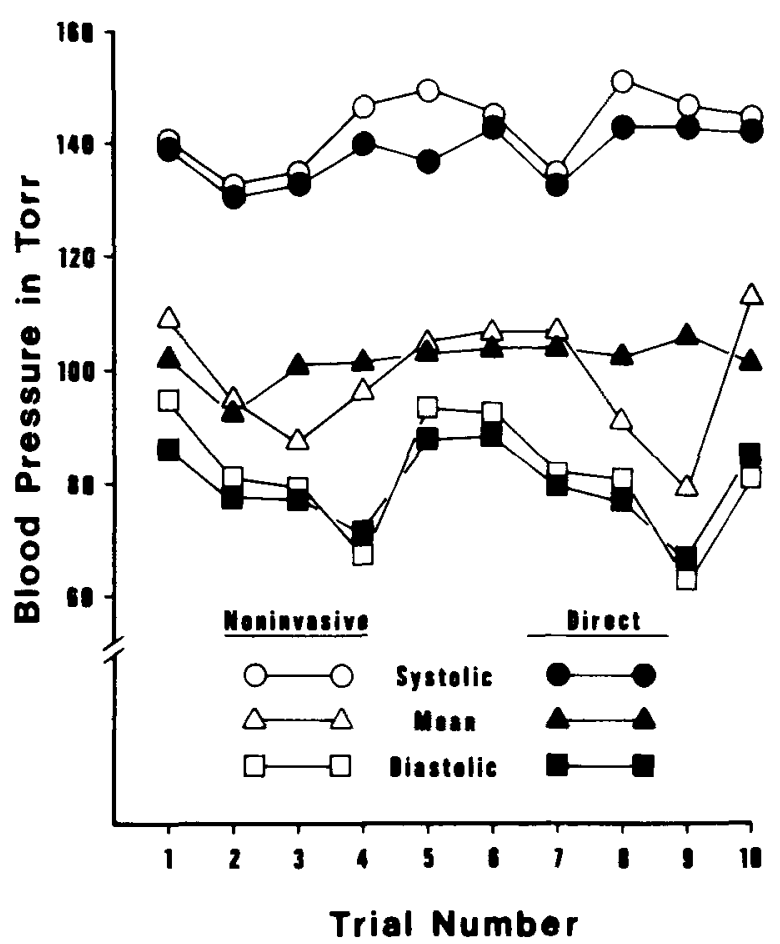

Figure 3. Simultaneous noninvasive and direct measurements of systolic, diastolic, and mean arterial BP in a conscious, trained baboon.

also tends to supress the effects of an occasional aberrant reading.

Examination of the direct recordings from one tethered baboon revealed an abrupt but transitory decline in BP immediately before some of the noninvasive trials. The 10- to 15-torr decreases appeared to be anticipatory responses to inflation of the occlusion cuff, as signaled by onset of the green ("apparatus operative") cue light. The animal's BP almost always returned to between-trial baseline levels in the interval between illumination of the cue light and the beginning of the cuff deflation phase when the noninvasive measurements were made. The other tethered baboon showed no changes in BP related to performance of the noninvasive measurement procedure.

\section{DISCUSSION}

The apparatus and animal training procedures described here permit frequent (e.g., daily) noninvasive measurements of BP in conscious, unrestrained nonhuman primates. The method provides accurate estimates of systolic, diastolic, and mean arterial pressure in a single measurement, and simultaneous computation of heart rate also is possible, based on interpulse interval data. By eliminating the need for anesthetics or physical restraint, the method avoids two major sources of confounding in the determination of BP values. Another unique and valuable feature of this completely automated method is that it does not require the presence of a human observer, an obvious advantage in behavioral and cardiovascular studies that seek to avoid spurious alterations in BP caused by extraneous stimuli or by potentially stressful environmental events.

By making the apparatus available at the animal's home cage, the time-consuming and sometimes dangerous problems associated with handling and transporting large nonhuman primates are eliminated. Use of the oscillometric approach also eliminates the need for shaving, depilation, or ultrasonic coupling gels.

Training baboons to participate in the $\mathrm{BP}$ measurement procedure involves straightforward operant conditioning methods, does not require an excessive number of sessions, and can be largely automated. The portability of the cage-mounted equipment makes possible training or testing of many animals each day. Finally, our experience indicates that, in the absence of competing or interfering tasks, many weeks can intervene between sessions with little or no deterioration of a trained animal's proficiency or willingness to perform the BP measurement procedure.

\section{REFERENCES}

BARD, P. (Ed.). Medical physiology. St. Louis: Mosby, 1956. Best, C. H., \& TAYLoR, N. B. The physiological basis of medical practice (7th ed.). Baltimore: Williams \& Wilkins, 1961.

BYRD, L. D. A tethering system for direct measurement of cardiovascular function in the caged baboon. American Journal of Physiology, 1979, 236, H775-H779.

DeitenBeck, W. H. A unique noninvasive technique for measuring the blood pressure of the neonate. Medical Instrumentation, 1977, 2, 54-55.

Flack, M., Hill, L., \& McQueEn, J. The measurement of arterial pressure in man: I. The auditory method. Proceedings of the Royal Society of London, B, Biological Science, 1915, 88, 508-536.

Geddes, L. S., Moore, A. G., Garner, H., Rosborough, J., Ross, J., \& AmEND, J. The indirect measurement of mean blood pressure in the horse. Southwest Veterinarian, 1970, 23, 289-294

Gi.fy, P.. \& Gomez, D. M. La détermination des pressions moyenne et minima par la méthode oscillométrique. Press Médicale, 1931, 39, 284-286.

Kirkendal.l, W. M., Burton, A. C., Epstein, F. H., \& Freis, E. D. Recommendations for human blood pressure determination by sphygmomanometers. Circulation, 1967, 36, 980-988.

LOONEY, J., Jr. Blood pressure by oscillometry. Medical Electronics, 1978, 9, 57-63.

McMahan, C. A., Wigodsky, H. S., \& Moore, G. T. Direct and indirect blood pressure measurements in baboons. Journal of Applied Physiology, 1976, 41, 806-809.

Mitchell., D. S., Peel, H. H., Wigodsky, H. S., \& Morris, M. D. Noninvasive oscillometric measurement of blood pressure in baboons (Papio cynocephalus). Laboratory Animal Science, 1980, 30, 666-672.

Pachon, V., \& Fabre, R. Sur le critère de la pression minima dans la méthode oscillométrique. Cômpte Rendu des Séances de la Société de Biologie Paris, 1921, 84, 871-874.

Posey, J. A., Grider, L. A., Williams, H., \& Moorf, A. G. 
The meaning of the point of maximum oscillations in cuff pressure in the indirect measurement of blood pressure: Part I. Cardiovascular Research Center Bulletin, 1969, 8, 15-25.

RAMSEY, M. Noninvasive device for automatic measurement of mean arterial pressure in adults and premature neonates. Proceedings of the Association for the Advancement of Medical Instrumentation. San Francisco: 1977.

Selkurt, E. E. Peripheral blood pressures and pulses: Venous pressure and venous return. In E. E. Selkurt (Ed.), Physiology (2nd ed.). Boston: Little, Brown, 1966.

Smith, C. D., \& Ansevin, A. Blood pressure of the normal rhesus monkey. Proceedings of the Society for Experimental Biology and Medicine, 1957, 96, 428-432.

(Received for publication October 2, 1980; accepted October 13, 1980.) 